\title{
Multiple Sclerosis in a 23 Years Old Woman Treated by Venous Angioplasty for Chronic Cerebrospinal Venous Insufficiency: A DNA Study by Flow Cytometry
}

Kalodimou VE ${ }^{1 *}$, Charalampopoulos $\mathbf{G}^{2}$, Bekos $\mathrm{V}^{3}$, Takis $\mathrm{K}^{4}$, Ghiatas $\mathrm{A}^{5}$, Liasis $\mathrm{N}^{2}$ and Tsamopoulos $\mathrm{NG}^{6}$

${ }^{1}$ IASO Maternity \& Research Hospital - Department of Flow Cytometry-Research \& Regenerative Medicine, Greece

${ }^{2}$ Medical Director of Euromedic Greece/ Evroiatriki Psychikou, Greece

${ }^{3} \mathrm{Naval}$ and Veterans Hospital of Athens - ICU, Greece

${ }^{4}$ Mediterraneo Hospital - Department of Neurology, Greece

5IASO Maternity \& Research Hospital - Department of Radiology, Greece

${ }^{6}$ Mediterraneo Hospital - Department of Interventional Neuroradiology, Greece

\begin{abstract}
We present a 23 years old Caucasian female patient with multiple sclerosis and Chronic Cerebrospinal Venous Insufficiency, treated by venous angioplasty of the extracranial veins. The presence of Chronic Cerebrospinal Venous Insufficiency (CCSVI) was detected by a sonogram of both Internal Jugular and Vertebral Veins with assessing grey scale appearance, color Doppler flow and transcranial sonogram of the deep cerebral veins using Quality Doppler Processing Technology (QDP). The genetic analyses disclosed aneuploidy DNA content by flow cytometry analysis. A Magnetic Resonance follow up is recommended to patients and their first degree relatives.
\end{abstract}

Keywords: Chronic cerebrospinal venous isufficiency; Multiple sclerosis; Aneuploidy DNA; Flow cytometry

Abbreviation: CCSVI: Chronic Cerebrospinal Venous Insufficiency; QDP: Quality Doppler Processing Technology; MS: Multiple Sclerosis; IJV: Internal Jugular Vein

\section{Background}

Multiple Sclerosis (MS) is an inflammatory demyelinating disease of the Central Nervous System (CNS). Subjects 20-40 years old are affected in a relapsing-remitting, secondary progressive or primary progressive pattern. Although the clinical course of the disease is variable, it usually leads to progressive deterioration and severe disability [1]. Both genetic and environmental factors have been implicated in the pathogenesis of MS [2]. The precise cause remains largely unknown, but there is evidence of a myelin-antigen-specific, Th1-mediated immune process resulting in macrophage-mediated demyelination, axonal loss and gliosis [3]. In the last years, a "vascular hypothesis" initially proposed by Charcot [4] has re-emerged, causing heated debates among physicians and huge expectations among patients. Zamboni et al. [5] combined transcranial and extracranial color Doppler ultrasonography to examine internal jugular, vertebral, deep cerebral, and azygos veins and evaluate 5 (five) parameters that assess venous blood flow and vessel anatomy [6]. A dramatic correlation between MS and the presence of 2 or more of the 5 criteria of venous outflow abnormalities was reported. This led the authors to a highly criticized hypothesis that chronically impaired venous drainage of the CNS (termed "Chronic Cerebrospinal Venous Insufficiency", CCSVI) may trigger the initiation of an inflammatory process leading to MS $[7,8]$. Chronic Cerebrospinal Venous Insufficiency (CCSVI) is described as a state of impaired venous brain drainage due to obstructions in the Internal Jugular Veins (IJVs), the vertebral veins, and the azygos vein, causing changes in the normal intra and extracranial haemodynamics and development of bypassing collaterals. Accumulating data that the obstructions in these veins are associated with developmental abnormalities led to inclusion of CCSVI in the classification of venous malformations by the International Union of Phlebology [9]. The CCSVI syndrome is associated with neurodegenerative disorders. Endovascular therapy appears to be a safe and reliable method for treating CCSVI $[10,11]$. The high incidence of recurrence of the MS in our case highlights the necessity of the genetic profile analysis in the patients and their first degree relatives.

\section{Case Presentation}

In a 23 years old Caucasian woman who was diagnosed 2 years ago with multiple sclerosis after an episode of left optic neuritis developed and treated with steroids with recovery of her vision. Head MRI revealed 6 lesions, with normal C-spine and T-spine MRI's. She decided not to take interferons.

On November 2012 according to the internationally recommended
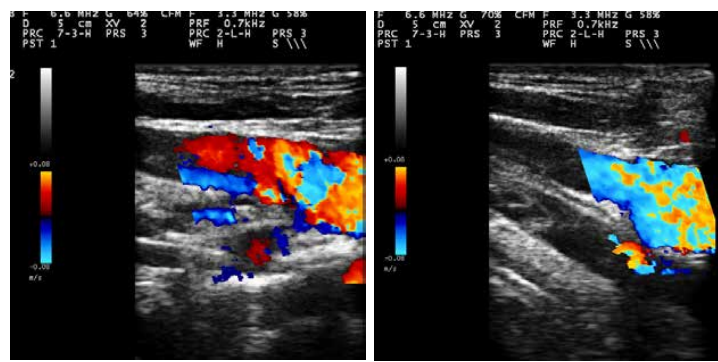

Figure 1: Patient - On the left reflux on the Internal Jugular Vein, Pre- procedure at Nov 2012. On the right NO Reflux, Post procedure at Jan 13.

*Corresponding author: Kalodimou VE, Flow Cytometry-Research \& Regenerative Medicine Department, IASO-Maternity \& Research Hospital, Kifisias Avenue 37-39, 151 25, Marousi, Athens, Greece, Tel: 0030-210-618-5203; Fax: 0030-210-610-5711; E-mail: kalodimou@yahoo.gr

Received June 21, 2013; Accepted July 07, 2013; Published July 11, 2013

Citation: Kalodimou VE, Charalampopoulos G, Bekos V, Takis K, Ghiatas A, et al (2013) Multiple Sclerosis in a 23 Years Old Woman Treated by Venous Angioplasty for Chronic Cerebrospinal Venous Insufficiency: A DNA Study by Flow Cytometry. Cardiol Pharmacol 2: 110. doi:10.4172/2329-6607.1000110

Copyright: (c) 2013 Kalodimou VE, et al. This is an open-access article distributed under the terms of the Creative Commons Attribution License, which permits unrestricted use, distribution, and reproduction in any medium, provided the original author and source are credited. 
protocol of CCSVI for diagnostic purpose, she had digital selective venography and angioplasty of the azygos vein and both internal jugular veins. The presence of CCSVI was detected by a sonogram of both internal jugular veins assessing with grey scale appearance and color Doppler flow, transcranial sonogram of the deep cerebral veins using Quality Doppler Processing Technology (QDP) (Figure 1).

Our protocol for endovascular treatment of CCSVI included balloon angioplasty in the IJVs and the azygos vein with the aim of restoring blood flow, not necessarily achieving a perfect angiographic result. The protocol for endovascular treatment of CCSVI was established with the approval of our Institutional Review Board (I.R.B). Endovascular treatment of CCSVI was performed under sedation, by means of balloon angioplasty (PTA).

In preparation for venography, percutaneous access of the left femoral vein was obtained and a short $8 \mathrm{Fr}$ introducer was used. Venography was performed with a $5 \mathrm{Fr}$ diagnostic catheter. For balloon dilatation an exchange guide wire passed via the diagnostic catheter through the stenosis for delivery of semi-compliant angioplasty balloon to the references vessels. A postangioplasty venogram was performed after every angioplasty. After achieving optimal results on the functionally significant lesions and performing final angiography, the guidewire and the introducer was removed and homeostasis was established by manual compression.

After the procedure her concentration and memory improved significantly, the numbness she felt in her arms and hands disappeared and her stamina also improved. Six months later after an episode of left tendonitis in her foot developed, she started having again numbness in her arms. These symptoms resolved in a few weeks. Presently the patient is totally asymptomatic.
Blood samples were analyzed on a Cytomics FC 500 flow cytometer (Beckman Coulter), set up with an argon-ion laser emitting at $488 \mathrm{~nm}$ (400 m W) for aneuploidy. A minimum number of 17.000 nuclei from the sample were analyzed. The data were displayed as histograms. The flow cytometry analysis revealed an aneuploid DNA content in blood sample between the father and the daughter of the family and not the mother (Figure 2).

\section{Discussion}

A sonogram of both internal jugular veins with assessing grey scale appearance and color Doppler flow, transcranial sonogram of the deep cerebral veins using Quality Doppler Processing Technology ( QDP) was performed to the mother 50 years old and the father 62 years old of the patient. Although none of them had 2 of the 5 sonographic diagnostic criteria for CCSVI: (1) Reflux in the internal jugular and/or vertebral veins, (2) reflux in the deep cerebral veins, (3) evidence of at least $50 \%$ stenosis of the internal jugular vein(s), (4) no flow detected in the internal jugular and/or vertebral vein(s)and (5) impaired postural flow pattern in the internal jugular or vertebral vein(s)' the mother revealed a septum at the right internal jugular vein and the father revealed reflux at the right internal jugular vein (Figure 3).

The performed genetic analysis of our patient family disclosed an aneuploid DNA content between the father and the daughter $(S=13.9 \%$ \& $\mathrm{G} 2=0 \%$ and $\mathrm{S}=0.8 \%$ \& $\mathrm{G} 2=0.6 \%$ respectively), with a $70.5 \%$ of cells in the DNA synthetic phase confirming the above assumption (Figure 2).

In this case report we try to investigate the possible prognostic value of ploidy in humans and the disruptions occurring inside the cell cycle with flow cytomety as a diagnostic tool. Flow cytometry enables rapid quantification of DNA content of individual cells, and the cellular DNA content provides useful information about the ploidy, expressing the

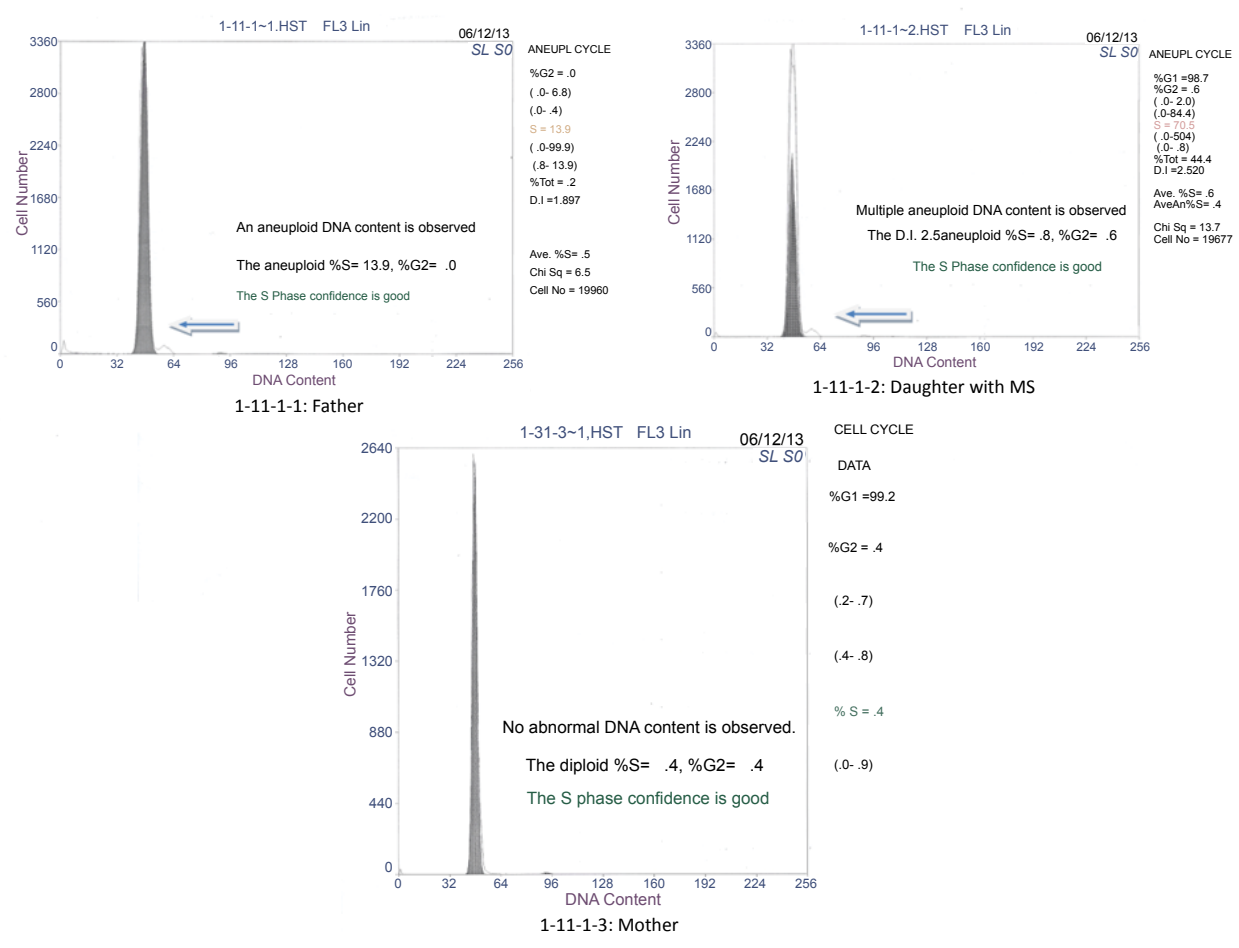

Figure 2: An aneuploid DNA (Diploid) content of revealed after Flow Cytometry analysis between the family. 
Citation: Kalodimou VE, Charalampopoulos G, Bekos V, Takis K, Ghiatas A, et al. (2013) Multiple Sclerosis in a 23 Years Old Woman Treated by Venous Angioplasty for Chronic Cerebrospinal Venous Insufficiency: A DNA Study by Flow Cytometry. Cardiol Pharmacol 2: 110. doi:10.4172/23296607.1000110

Page 3 of 3

modal DNA value, and the proliferative activity in a tissue. The ability of flow cytometry to estimate cellular DNA content is based on the measurement of fluorescence from dyes which bind in a stoichiometric manner to DNA [12].

As the DNA content is duplicated prior to cell division, mathematical models have been derived which can estimate the percentage of cells in different phases of the cell cycle. The performed genetic analysis between the family members (father \& daughter) disclosed an aneuploid DNA content, with a $13.9 \%$ (father) \& $70.5 \%$ (daughter) of cells in the DNA synthetic phase confirming the above assumption.

Using flow cytometry for DNA analysis between family members with genetically linked diseases provides fast results, permits multiparameter analysis correlating DNA content with antigen expression, and also provides sensitivity for detecting near-diploid aneuploid peaks [13]

\section{Conclusion}

Multiple Sclerosis (MS) is an inflammatory demyelinating disease of the Central Nervous System (CNS). Subjects 20-40 years old are affected in a relapsing-remitting, secondary progressive or primary progressive pattern. Although the clinical course of the disease is variable, it usually leads to progressive deterioration and severe disability.

Venus angioplasty of the extra cranial veins in the attempt to treat the CCSVI Syndrome in MS patients has generated conflicted results [14]. Published studies so far displays remarkable heterogeneity regarding study protocols requirements, making evidence based comparison difficult due to lack of a standardized methodology and procedural techniques [15]. We are waiting the results of the ongoing trials.

In cases like this, we consider that a genetic profile analysis should be performed to patients and their first degree relatives for more efficient therapies and follow ups of the disease. Flow Cytometry as an analysis method is a rapid, sensitive accurate and efficient for the genetic analysis and the progress of the disease.

\section{Consent}

The risks and benefits of endovascular treatment for CCSVI were explained to the patient and she agreed to endovascular treatment and signed an informed consent form prior to hospitalization.

\section{Disclosure Statement and Competing Interests}

All authors have made substantive contributions to the study, and

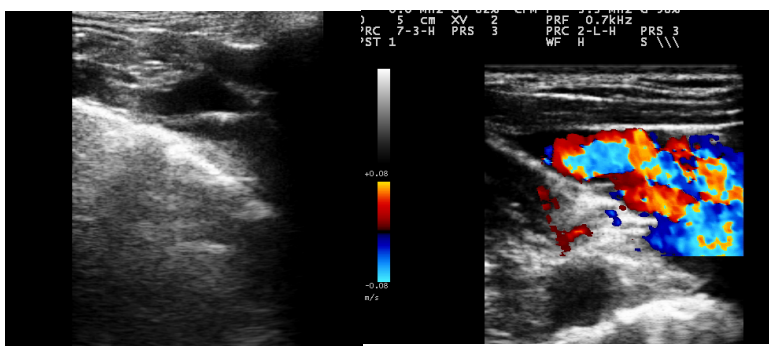

Figure 3: On the left, mother revealed a septum on the Right Internal Jugular Vein. On the right, father revealed a reflux at his Right Internal Jugular vein. all authors endorse the conclusion. None of the authors has a conflict of interest.

\section{Authors' Contribution}

Tsamopoulos NG, Charalampopoulos G, Bekos V, Takis K, Ghiatas A, Liasis $\mathrm{N}$ were involved with the patient's management. Kalodimou VE is the head of Flow Cytometry-Research \& Regenerative Medicine Department, IASO Maternity Hospital, who carried out the DNA analysis.

Tsamopoulos NG is the Head of Interventional Neuroradiology Department at Mediterraneo Hospital. Bekos V is the Chief of ICU at Naval \& Veterans Hospital of Athens. Liasis N is the Medical Director of Euromedic Greece/ Evroiatriki Psychikou. Ghiatas A is the Head of Radiology Department at IASO Maternity Hospital. Takis K is the Head of the Neurology Department at Mediterraneo Hospital. Kalodimou VE and Tsamopoulos NG prepared, wrote and revised the article. All authors read and approved the final manuscript.

\section{References}

1. Weinshenker BG, Bass B, Rice GP, Noseworthy J, Carriere W, et al. (1989) The natural history of multiple sclerosis: a geographically based study. 2 . Predictive value of the early clinical course. Brain 112: 1419-1428.

2. Moore GR (2010) Current concepts in the neuropathology and pathogenesis of multiple sclerosis. Can J Neurol Sci 37: S5-15.

3. Ontaneda D, Hyland M, Cohen JA (2012) Multiple sclerosis: new insights in pathogenesis and novel therapeutics. Annu Rev Med 63: 389-404.

4. Charcot J (1868) Histology of "sclerose en plaque" (in French). Gazette Hosp (Paris) 1868: 554-566.

5. Zamboni P, Menegatti E, Galeotti R, Malagoni AM, Tacconi G, et al. (2009) The value of cerebral Doppler venous haemodynamics in the assessment of multiple sclerosis. J Neurol Sci 282: 21-27.

6. Nicolaides AN, Morovic S, Menegatti E, Viselner G, Zamboni P (2011) Screening for chronic cerebrospinal venous insufficiency (CCSVI) using ultrasound: recommendations for a protocol. Funct Neurol 26: 229-248.

7. Zamboni $P$ (2006) The big idea: iron-dependent inflammation in venous disease and proposed parallels in multiple sclerosis. J R Soc Med 99: 589-593.

8. Zamboni P, Galeotti R, Menegatti E, Malagoni AM, Tacconi G, et al. (2009) Chronic cerebrospinal venous insufficiency in patients with multiple sclerosis. $J$ Neurol Neurosurg Psychiatry 80: 392-399.

9. Lee BB, Bergan J, Gloviczki P, Laredo J, Loose DA, et al. (2009) Diagnosis and treatment of venous malformations. Consensus document of the International Union of Phlebology (IUP)-2009. Int Angiol 28: 434-451.

10. Petrov I, Grozdinski L, Kaninski G, Iliev N, Iloska M, et al. (2011) Safety profile of endovascular treatment for chronic cerebrospinal venous insufficiency in patients with multiple sclerosis. J Endovasc Ther 18: 314-323.

11. Zamboni P, Bertolotto A, Boldrini P, Cenni P, D'Alessandro R, et al (2012) Efficacy and safety of venous angioplasty of the extracranial veins for multiple sclerosis. Brave dreams study (brain venous drainage exploited against multiple sclerosis): study protocol for a randomized controlled trial. Trials 13 183.

12. Dressler LG, Seamer LC (1994) Controls, standards, and histogram interpretation in DNA flow cytometry. Methods Cell Biol 41: 241-262.

13. Kalodimou VE (2013) Basic principles in flow cytometry. American Association of Blood Banks.

14. Ghezzi A, Comi G, Federico A (2011) Chronic cerebro-spinal venous insufficiency (CCSVI) and multiple sclerosis. Neurol Sci 32: 17-21.

15. Thapar A, Lane T, Nicholas R, Friede T, Ellis M, et al. (2011) Systematic review of sonographic chronic cerebrospinal venous insufficiency findings in multiple sclerosis. Phlebology 26: 319-325. 